\title{
Correlación del grado de percepción y cultura de seguridad del paciente en una Institución de tercer nivel 2015- 2019
}

Correlation between the Degree of Perception and Culture on Patient Safety in a Tertiary Hospital between 2015-2019

Correlação do grau de percepção e cultura da segurança do paciente em um hospital de nível terciário entre 2015-2019

Como citar este artículo: Jaimes-Valencia Mary Luz, Alvarado-Alvarado Andrés Leonardo, Mejía-Arciniegas Claudia Nathaly, López-Galán Angie Valentina, Mancilla-Jiménez Vivian Angélica, Padilla-García Clara Inés. Correlación del grado de percepción y cultura de seguridad del paciente en una Institución de tercer nivel 2015-2019 Revista Cuidarte. 2021;12(1):e1092. http://dx.doi.org/10.15649/cuidarte.1092

Revista Cuidarte

doi. Rev Cuid. Ene. - Abril. 2021; 12(1): e1092 http://dx.doi.org/10.15649/cuidarte.1092

\section{E-ISSN: 2346-3414}

(1) Mary Luz Jaimes Valencia' ${ }^{1}$

(1) Andrés Leonardo Alvarado Alvarado $^{2}$

(1) Claudia Nathaly Mejía Arciniegas ${ }^{3}$

(1) Angie Valentina López Galán ${ }^{4}$

(1) Vivian Angélica Mancilla Jiménez

(1) Clara Inés Padilla García ${ }^{6}$

1 Enfermera, Magíster en Enfermería con Énfasis en Salud Familiar, Doctorado en enfermería Práctica y Educación, Docente Universidad Autónoma de Bucaramanga, Santander, Colombia.

E-mail: mjaimes239@unab.edu.co.

2 Enfermero, Servicio de Cuidado Crítico Adulto/Unidad de trasplante ECMO; Fundación Cardiovascular de Colombia; Floridablanca, Santander, Colombia. E-mail:

aalvarado510@unab.edu.co

3 Enfermera, Servicio de hospitalización, Fundación Oftalmológica de Santander, Santander, Colombia. E-mail: cmejia700@unab.edu.co.

4 Enfermera, Servicio de investigación, Fundación Cardioinfantil - Instituto de Cardiología, Bogotá, Cundinamarca, Colombia.

E-mail: alopez747@unab.edu.co.

5 Enfermera, Especialista en Gerencia de Servicios de Salud, Especialista en Seguridad y Salud en el Trabajo, Coordinadora de Calidad de M\&S Solutions, Santander, Colombia. E-mail: vivianmancilla@gmail.com.

6 Enfermera, Especialista en Enfermería en Cuidado Crítico, Magíster en Enfermería con Énfasis en Cuidado Cardiovascular, Docente Universidad Industrial de Santander, Bucaramanga, Santander, Colombia. E-mail: cipadiga@uis.edu.co

\section{Resumen}

Introducción: La seguridad del paciente es considerado como un patrón integrado de comportamiento individual y organizacional, basado en creencias y valores compartidos que continuamente busca minimizar el daño al paciente, que resulta de la atención. Objetivo: Analizar la correlación de percepción de cultura de seguridad del paciente, y grado de seguridad percibido en funcionarios de una Institución de Salud de tercer nivel de atención (2015-2017-2019). Materiales y Métodos: Estudio cuantitativo correlacional, con una muestra intencional derivada del registro de una base de datos de cultura de seguridad del paciente, con un total de 402 registros. La medición se realizó mediante el cuestionario Hospital Survey on Patient Safety Culture. Resultados: En los resultados globales el mayor porcentaje de encuestados correspondió al personal asistencial (73,4\%). El principal servicio asistencial es unidad de cuidado intensivo (18,2\%) y la mayor participación correspondió al rol de enfermeras y auxiliares de enfermería (45,7\%). Según las respuestas positivas para los datos globales se determina 2 de 12 dimensiones clasificadas como fortaleza en el componente de Aprendizaje organizacional (81,5\%) y Trabajo en equipo $(85,5 \%)$ y el grado de seguridad percibido con correlaciones ( $p$ : $0,000)$ que se identifican en seis dimensiones entre ellas notificación de eventos, y aprendizaje organizacional. Discusión: La correlación del grado de seguridad percibido con las dimensiones del cuestionario guarda similitud en especial la notificación de eventos. Conclusión: La seguridad del paciente y el grado de seguridad percibido guardan correspondencia en la medida que en las instituciones realizan intervenciones en diferentes componentes entre ellos la notificación de eventos y trabajo en equipo.

Palabras clave: Enfermería; Calidad de la Atención de Salud; Seguridad del Paciente; Cultura Organizacional; Personal de salud.
Recibido: 5 de diciembre de 2019

Aceptado: 18 de julio de 2020

Publicado: 12 de febrero de 2021 $\square *$ Correspondência Mary Luz Jaimes Valencia E-mail:mjaimes239@unab.edu.co. 


\section{Correlation between the Degree of Perception and Culture on Patient Safety in a Tertiary Hospital between 2015-2019}

\section{Abstract}

Introduction: Patient safety is defined as an integrated pattern of individual and organizational behavior based on shared beliefs and values that continuously seeks to minimize harm to patients. Objective: To analyze the correlation between the perception of patient safety culture and the degree of safety perceived by healthcare workers in a tertiary hospital in 2015, 2017 and 2019. Materials and Methods: A quantitative correlational study was conducted with a purposive sample of 402 records obtained from a patient safety culture database. The Hospital Survey on Patient Safety Culture was used for assessment. Results: The global results show that the highest percentage of respondents corresponded to healthcare workers (73.4\%). The main healthcare service provided is the intensive care unit (18.2\%) in which most participants were nurses (45.7\%). According to positive responses received for global data, 2 out of the 12 dimensions are classified as strengths in the Organizational Learning (81.5\%) and Teamwork (85.5\%) components, as well as the degree of safety perceived through correlations ( $\mathrm{p}: 0.000$ ) is found in five dimensions such as event notifications and organizational learning, among others. Discussion: The correlation between the perceived degree of safety and survey dimensions shows similarity, especially for event notifications. Conclusion: Patient safety and the perceived degree of safety are correlated as institutional interventions are made for different components, including organizational learning and teamwork.

Key words: Nursing; Quality of Health Care; Safety Patient; Organizational Culture, Health Personnel.

\section{Correlação do grau de percepção e cultura da segurança do paciente em um hospital de nível terciário entre 2015-2019}

\section{Resumen}

Introdução: A segurança dos pacientes é definida como um padrão integrado de comportamento individual e organizacional baseado em crenças e valores compartilhados que busca continuamente minimizar os danos aos pacientes. Objetivo: Analisar a correlação entre a percepção da cultura de segurança do paciente e o grau de segurança percebido pelos profissionais de saúde em um hospital de nível terciário em 2015, 2017 e 2019. Materiais e Métodos: Um estudo correlacional quantitativo foi realizado com uma amostra proposital de 402 registros obtidos de um banco de dados de cultura de segurança do paciente. A Pesquisa sobre Cultura de Segurança dos Pacientes em Hospitais foi utilizada para a avaliação. Resultados: Os resultados globais mostram que a maior porcentagem de entrevistados correspondeu a trabalhadores da área de saúde (73,4\%). O principal serviço de saúde prestado é a unidade de terapia intensiva $(18,2 \%)$ e a maioria dos participantes eram enfermeiros $(45,7 \%)$. De acordo com as respostas positivas recebidas para os dados globais, 2 das 12 dimensões são classificadas como pontos fortes nos componentes Aprendizagem Organizacional (81,5\%) e Trabalho em Equipe (85,5\%), assim como o grau de segurança percebido através de correlações (p: 0,000) é encontrado em cinco dimensões, tais como notificações de eventos e aprendizagem organizacional, entre outras. Discussão: A correlação do grau de segurança percebido com as dimensões da pesquisa é particularmente semelhante à notificação de eventos. Conclusão: $A$ segurança do paciente e o grau de segurança percebido são correspondentes à medida que são feitas intervenções institucionais para diferentes componentes, incluindo o aprendizado organizacional e o trabalho em equipe.

Palavras chaves: Enfermagem; Qualidade da Assistência à Saúde; Segurança do Paciente; Cultura Organizacional; Pessoal de Saúde. 


\section{Introducción}

El tema de seguridad en la atención de los pacientes ha sido motivo de preocupación de personajes como Hipócrates, y en las ultimas 2 décadas, los sistemas de salud han tenido un gran interés por este tema. Por otra parte, en 1999, se publicó el reporte "Errar es humano", informe que escandalizó al mundo, debido a que, en esta publicación de los Estados Unidos, se describió que alrededor de 98,000 personas morían cada año, como producto de errores en la atención médica para la década de los 90 . Siendo este reporte la piedra angular para el desarrollo del movimiento mundial por la seguridad de los pacientes ${ }^{1-5}$.

A través del tiempo se ha desarrollado distintos conceptos y definiciones para seguridad del paciente (SP), dadas por autores o por organizaciones internacionales, en el caso de este estudio, se adoptó la definición de la Sociedad Europea para la calidad de la atención en salud, que define la seguridad del paciente como "Un patrón integrado de comportamiento individual y organizacional, basado en creencias y valores compartidos que continuamente busca minimizar el daño al paciente, que puede resultar

“Un patrón integrado de comportamiento individual y organizacional, basado en creencias y valores compartidos que continuamente busca minimizar el daño al paciente, que puede resultar de los procesos de atención ${ }^{\prime \prime}$ de los procesos de atención" ${ }^{\prime 6}$.

Para la Organización Mundial de la Salud (OMS), la seguridad del paciente es un principio sanitario. Aunque, hay un cierto grado de peligrosidad y dificultad en el proceso de atención de salud; el mejoramiento continuo de la seguridad del paciente requiere un compromiso y esfuerzo complejo que abarca una amplia gama de acciones dirigidas hacia la mejora del desempeño, la gestión de la calidad, seguridad y los riesgos ambientales que intrínsecamente se ven plasmado en cada acción del personal de salud ${ }^{7-9}$.

Los eventos adversos, son indicadores potenciales en el entorno de la seguridad del paciente y es pertinente precisar que estos eventos pueden ser prevenibles y no prevenibles. En su mayoría, están relacionados con el ambiente y área clínica asistencial; y cabe recalcar que mientras exista un paciente se puede presentar un evento adverso y disminuir la calidad de la atención en salud. Debido a esto el objetivo en común para mejorar la cultura de seguridad del paciente es minimizar los errores relacionados con la atención en salud a nivel mundial ${ }^{10-11}$.

En Latinoamérica el primer estudio del problema de la seguridad fue el estudio IBEAS. Hecho en 5 países de la región el cual evidenció que, en estos países, en promedio 1 de cada 10 pacientes hospitalizados (10\%) sufren al menos un daño durante su atención ${ }^{12}$.

En Colombia, el estudio IBEAS, mostró una prevalencia de eventos adversos del 13,1\% en general, en el grupo de menores de 15 años del 27,3\% y durante la realización de un procedimiento del $27,7 \%$. Cabe recalcar que a diferencia de otros países la mayoría de hospitales participantes estuvieron acreditados ${ }^{12}$.

Durante todos estos años, millones de dólares se han invertido para continuar la investigación en la evidencia científica de las prácticas seguras, educación alrededor del tema, buscando disminuir cada vez más la posibilidad de tener eventos adversos prevenibles durante la atención 
médica, sin embargo, la alta prevalencia de reportes de eventos adversos a nivel mundial, la seguridad del paciente sigue siendo una prioridad global ${ }^{13-15}$.

En Colombia, los avances que se han desarrollado se destaca el Sistema Único de Acreditación en salud que para el año 2005 introdujo, como requisito para acreditación institucional, la exigencia de tener un sistema de reporte de eventos adversos, lo cual llevo voluntariamente a estas instituciones a diseñar un programa de seguridad del paciente. En 2008 el Ministerio de Salud en Colombia impulsó una Política y una guía de Seguridad del Paciente. Con fin de complementar la Política de Seguridad del Paciente el Ministerio de Salud desarrolló paquetes instruccionales para que las instituciones tuvieran directrices técnicas para su implementación práctica. Hoy, estos paquetes son actualizados con el propósito de ajustarlos a la mejor evidencia disponible en la actualidad ${ }^{16}$.

Respectivamente para al año 2010 el Ministerio de Salud de Colombia impulso una guía técnica definida como "Buenas prácticas para la seguridad del paciente en la atención en salud" en conjunto con un paquete instruccional que se define con el mismo título de la guía técnica, con el fin de fortalecer la Seguridad del Paciente, el Ministerio de Salud definió en estos documentos unas pautas que se desarrollan como indicadores directos de la seguridad del paciente. Estos indicadores se describen como:

- Gestión de eventos adversos.

- Reporte de eventos adversos.

- Indicadores que reporten y evalúen el impacto en caídas, infecciones, neumonías y otros aspectos clínicos que se desarrollen en el contexto de cuidado de la salud.

- Detección del ambiente físico.

- Vigilancia de eventos adversos de un prestador de salud, aseguradora, una red o una región ${ }^{16}$.

Estos indicadores como se mencionó anteriormente están relacionados con la seguridad del paciente y tienen un compromiso en la medición de la calidad clínica y administrativa de las instituciones prestadoras de servicios de salud ${ }^{17}$.

Respecto a la cultura de seguridad del paciente en Colombia, el Ministerio de Salud y Protección Social e instituciones, han desarrollado guías para la promoción de la cultura de seguridad del paciente, con el objetivo de identificar las características de los programas referentes a la descripción de la cultura organizacional, el clima laboral y la preparación del personal de la salud, respecto al afrontamiento y disminución del riesgo de presentar eventos adversos en los individuos $^{18,19}$.

En países como Estados Unidos, Australia, Reino Unido e Irlanda del norte, desarrollaron estudios de gran magnitud para el análisis de la seguridad del paciente, así mismo crearon diferentes sistemas enfocados a mejorar la Cultura de seguridad, como es el caso de Estados Unidos, donde distintas organizaciones e instituciones se unieron para formar las Organizaciones de Seguridad del Paciente (PSO de sus siglas en inglés), medida y acreditada por la Agencia de Investigación y la Calidad de la Atención Sanitaria (AHRQ de sus siglas en inglés), cuyo objetivo es aumentar la cultura de calidad de su modelo de salud ${ }^{20,21}$. En el caso de España se creó el proyecto SENECA, basado en el modelo Europeo de Excelencia Empresarial (EFQM de sus siglas en inglés) con el propósito de evaluar la calidad de los cuidados para la seguridad del paciente en los hospitales ${ }^{22}$. 
A nivel nacional, en Bogotá se evidencio la necesidad de estrategias basadas en la educación, aprovechando las fortalezas, para superar las falencias existentes, y revisar las condiciones laborales, que puedan ser una barrera para el fortalecimiento del programa de seguridad del paciente. En Santander, son escasos los estudios respecto a la evaluación de seguridad del paciente, pero, el estudio de Bravo, desarrollado en este departamento; pudo evidenciar que la seguridad del paciente se ve fortalecida por la comunicación, clima laboral y la buena cultura del reporte de eventos adversos, que logra identificar las falencias de las áreas clínicas y así mismo desarrollar planes de mejoramiento para los servicios de atención al paciente ${ }^{23}$. De esta manera se considera pertinente realizar un estudio que permita analizar la correlación de percepción de cultura de seguridad del paciente, y grado de seguridad percibido en funcionarios de una Institución de Salud de III nivel de atención reportado en los años de 2015, 2017 y 2019.

\section{Materiales y Métodos}

\section{Diseño: Estudio cuantitativo correlacional.}

Muestra: Corresponde a una muestra intencional derivada del registro de la base de datos de cultura de seguridad del paciente, de una institución de tercer nivel de atención en salud con sedes en Bucaramanga y Bogotá (Nororiente y centro Colombiano, respectivamente). Se obtuvo un total de 402 registros, divididos en tres años (2015-2017-2019), para el año 2015 se reportó un total de 172 registros procedentes de la sede de Bucaramanga y Bogotá, para el año 2017 se obtuvo un total de 131 registros procedentes de la sede de Bucaramanga y Bogotá; y para el año 2019 se reportó un total de 99 registros procedentes de la sede Bucaramanga. Los funcionarios enviaron mediante su usuario a la plataforma de registro clínico de la institución las respuestas a la evaluación de seguridad del paciente de manera electrónica. Cabe recalcar que el reporte y aplicación de la encuesta se realiza cada 2 años al personal nuevo y antiguo, de todas las áreas de atención, ya sea clínico o administrativo. De los 402 registros, hay funcionarios que han participado en el desarrollo de la encuesta en varios años.

Instrumento: La información fue recolectada de manera electrónica con el uso del instrumento institucional que se usa para valorar la cultura de seguridad del paciente y que corresponde al instrumento "Cuestionario sobre Seguridad de los Pacientes en Hospitales", versión Española adaptada del Hospital Survey on Patient Safety Culture, de la Agencia de Investigación y la Calidad de la Atención Sanitaria $(\mathrm{AHRQ})^{24}$, En el contexto Colombiano se ha desarrollado la validación cultural de este instrumento obteniendo datos de fiabilidad mediante el a Cronbach entre 0,64 hasta $0,88^{25}$. El cuestionario utilizado en este estudio corresponde al de origen español que es el que institucionalmente se viene usando desde el año 2015 a la fecha y consta de 62 ítems, clasificado en una escala tipo Likert de 5 puntos, los ítems 1 a 33: 1 (muy en desacuerdo), 2 (en desacuerdo), 3 (ni de acuerdo ni en desacuerdo), 4 (de acuerdo), 5 (muy de acuerdo) y los ítems 34 a 51: 1 (Nunca), 2 (Raramente), 3 (A veces), 4 (Casi siempre), 5 (Siempre) ${ }^{26,27}$. Según el instrumento, para cada dimensión, respectivamente a la puntuación de frecuencia (porcentaje), se puede clasificar en tres componentes: Fortaleza, que es la puntuación de respuestas positivas $\geq 75 \%$, Oportunidad de mejora con puntuación de respuestas positivas $\geq 50 \%$ hasta $74.9 \%$ y Debilidad con puntuación de respuestas positivas $<50 \%{ }^{28}$.

El cuestionario consta de 4 secciones: A: su servicio/unidad. B: su hospital. C: comunicación en su servicio/unidad. D: información complementaria (datos generales de la institución y un ítem 
que valora el grado de seguridad percibida)

\section{El cuestionario comprende 12 dimensiones que permiten evaluar la cultura de seguridad:}

- Dimensión 1: Frecuencia de eventos notificados (3 ítems).

- Dimensión 2: Percepción de seguridad (4 ítems).

- Dimensión 3: Expectativas y acciones de la dirección/ supervisión de la Unidad/ Servicio que favorecen la seguridad (4 ítems).

- Dimensión 4: Aprendizaje organizacional/mejora continua (3 ítems).

- Dimensión 5: Trabajo en equipo en la Unidad/Servicio (4 ítems).

- Dimensión 6: Franqueza en la comunicación (3 ítems).

- Dimensión 7: Feed-back y comunicación sobre errores (3 ítems).

- Dimensión 8: Respuesta no punitiva a los errores (3 ítems).

- Dimensión 9: Dotación del personal (4 ítems).

- Dimensión 10: Apoyo de la gerencia del hospital en la seguridad del paciente (3 ítems).

- Dimensión 11:Trabajo en equipo entre unidades (4 ítems).

- Dimensión 12: Problemas en cambios de turno y transiciones entre servicios/ unidades (4 ítems $)^{26}$.

Consideraciones éticas: Este estudio contempló los aspectos éticos establecidos en la resolución 8430 de 1993 del Ministerio de Salud de Colombia, la cual regula la investigación en seres humanos, la declaración de Helsinki que determina los principios éticos de la investigación médica en los seres humanos y la Ley 911 de 2004 la cual dicta la disposición en materia de responsabilidad deontológica para el ejercicio de la Profesión de Enfermería, se clasificó este estudio como de riesgo mínimo. Se obtuvo el permiso institucional para acceder a la base de datos de los registros de evaluación de seguridad del paciente y el permiso de la coordinadora de la sede de Bucaramanga del comité de Seguridad del Paciente, Calidad y Vigilancia Epidemiológica. Para la protección de los datos de identificación de los participantes, se otorgó un código numérico consecutivo entero a cada registro permitiendo el anonimato de los mismos.

Sistematización y análisis de datos: La sistematización de los datos, se realizó en una tabla de Excel, donde se adjuntaron y codificaron los datos obtenidos, posteriormente se exportaron al programa estadístico Statistical Package for Social Science (SPSS) versión 24, donde se desarrollaron los análisis descriptivos, frecuencias, media; y análisis de correlación medido a través de la significancia bilateral del coeficiente de correlación de Pearson. La correlación se implementó entre la asociación de la pregunta número 52 (grado de seguridad percibida) como variable independiente y las 12 dimensiones del cuestionario.

\section{Resultados}

Según el análisis de las características sociodemográficas, se evidencio que hubo mayor participación del personal asistencial, áreas clínicas de Unidad de cuidado intensivo y Hemodinamia, Respecto al rol de los participantes el mayor porcentaje corresponde al rol de enfermería (auxiliar y profesional); mayor porcentaje de trabajo semanalmente de 20 a 48 horas y antigüedad en la institución de 3 a 5 años (ver tabla 1) 
Tabla 1. Características de la población a estudio

\begin{tabular}{|c|c|c|c|c|c|c|c|c|}
\hline \multirow[t]{2}{*}{ Características } & \multicolumn{2}{|c|}{$\begin{array}{c}2015 \\
\text { (n: 172) }\end{array}$} & \multicolumn{2}{|c|}{$\begin{array}{c}2017 \\
\text { (n: 131) }\end{array}$} & \multicolumn{2}{|c|}{$\begin{array}{c}2019 \\
\text { (n: 99) }\end{array}$} & \multicolumn{2}{|c|}{$\begin{array}{l}\text { Global } \\
\text { (n: 402) }\end{array}$} \\
\hline & $\mathbf{n}$ & $\%$ & $\mathrm{n}$ & $\%$ & $\mathrm{n}$ & $\%$ & $\mathrm{n}$ & $\%$ \\
\hline \multicolumn{9}{|l|}{ Distribución de la muestra } \\
\hline Personal Administrativo & 28 & 16,3 & 45 & 34,3 & 46 & 46,5 & 119 & 26,6 \\
\hline Personal Asistencial & 144 & 83,7 & 86 & 65,6 & 53 & 53,5 & 283 & 73,4 \\
\hline \multicolumn{9}{|c|}{ Principal servicio o unidad de trabajo en la institución (P 60) } \\
\hline Anestesia y cirugía cardiovascular & 18 & 10,5 & 7 & 5,3 & 7 & 7,1 & 32 & 8,0 \\
\hline Pediatría & 1 & 0,6 & 1 & 0,8 & 2 & 2,0 & 4 & 1,0 \\
\hline Área administrativa & 28 & 16,3 & 45 & 34,4 & 46 & 46,5 & 119 & 29,6 \\
\hline Consulta externa & 2 & 1,2 & 2 & 1,5 & 1 & 1,0 & 5 & 1,2 \\
\hline Farmacia & 6 & 3,5 & 3 & 2,3 & 7 & 7,0 & 16 & 4,0 \\
\hline Hemodinamia y Electrofisiología & 31 & 18,0 & 14 & 10,7 & 16 & 16,2 & 61 & 15,2 \\
\hline Investigación & 2 & 1,2 & 0 & 0 & 2 & 2,0 & 4 & 1.0 \\
\hline Laboratorio & 3 & 1,7 & 5 & 3,8 & 0 & 0,0 & 8 & 2,0 \\
\hline Métodos Diagnósticos no Invasivos & 16 & 9,3 & 11 & 8,4 & 16 & 16,2 & 43 & 10,7 \\
\hline Unidad de cuidados intensivos & 40 & 23,3 & 33 & 25,2 & 0 & 0,0 & 73 & 18,2 \\
\hline Otro & 25 & 14,5 & 10 & 7,6 & 2 & 2,0 & 37 & 9,2 \\
\hline \multicolumn{9}{|l|}{ Rol en el puesto de trabajo (P 61) } \\
\hline Administrativo & 26 & 15,1 & 41 & 31,3 & 44 & 44,4 & 111 & 27,6 \\
\hline Auxiliar de enfermería & 48 & 27,9 & 26 & 19,8 & 19 & 19,2 & 93 & 23,1 \\
\hline Auxiliar de farmacia & 4 & 2,3 & 3 & 2,3 & 5 & 5,1 & 12 & 3,0 \\
\hline Bacterióloga/o & 3 & 1,7 & 7 & 5,3 & 0 & 0,0 & 10 & 2,5 \\
\hline Enfermería & 49 & 28,5 & 27 & 20,6 & 15 & 15,2 & 91 & 22,6 \\
\hline Instrumentación & 7 & 4,1 & 2 & 1,5 & 3 & 3,0 & 12 & 3,0 \\
\hline Medicina & 11 & 6,4 & 6 & 4,6 & 5 & 5,0 & 23 & 5,7 \\
\hline Químico farmacéutico & 3 & 1,7 & 0 & 0 & 1 & 1,0 & 4 & 1,0 \\
\hline Atención al usuario & 0 & 0,0 & 18 & 13,7 & 7 & 7,1 & 25 & 6,2 \\
\hline Otro & 21 & 12,2 & 0 & 0,0 & 0 & 0,0 & 21 & 5,2 \\
\hline \multicolumn{9}{|c|}{ Horas por semana trabajadas habitualmente en el servicio (P 56) } \\
\hline Menos $20 \mathrm{H}$ & 4 & 2,3 & 0 & 0,0 & 0 & 0,0 & 4 & 0,9 \\
\hline $20-48 \mathrm{H}$ & 111 & 64,5 & 99 & 75,6 & 73 & 73,7 & 283 & 70,3 \\
\hline 48H o más & 57 & 33,1 & 32 & 24,4 & 26 & 26,3 & 115 & 28,6 \\
\hline \multicolumn{9}{|c|}{ Años antigüedad en el servicio (P 55) } \\
\hline Menos 1 año & 58 & 33,7 & 23 & 17,6 & 13 & 13,1 & 94 & 23,4 \\
\hline 1 - 2 años & 33 & 19,2 & 24 & 18,3 & 9 & 9,1 & 66 & 16,4 \\
\hline $3-5$ años & 49 & 28,5 & 56 & 42,7 & 33 & 33,3 & 138 & 34,3 \\
\hline 6-15 años & 22 & 12,8 & 26 & 19,9 & 42 & 32.4 & 90 & 22,4 \\
\hline 16-20 años & 2 & 1,2 & 2 & 1,5 & 1 & 1,0 & 5 & 1,2 \\
\hline 21 años o más & 8 & 4,7 & 0 & 0 & 1 & 1,0 & 9 & 2,2 \\
\hline
\end{tabular}

P60: Equivale a la pregunta número 60 del instrumento. P61: Equivale a la pregunta número 61 del instrumento. P56: Equivale a la pregunta número 56 del instrumento. P55: Equivale a la pregunta número 55 del instrumento.

Fuente: Elaboración propia. 


\section{De seguridad del paciente}

Según el análisis de la cultura de seguridad del paciente con base en los parámetros del cuestionario Hospital Survey on Patient Safety Culture, se obtuvo los porcentajes de respuestas clasificadas como positivas, neutras y negativas. Describiendo de esta manera el indicador de fortaleza con las respuestas positivas $\geq 75 \%$, tomando esta clasificación para el análisis global, se observa un total de 2 dimensiones de 12 catalogadas como fortaleza, en el componente de Aprendizaje organizacional/mejora continua (81,5\%) y Trabajo en equipo en el Servicio (85,5\%). En la comparación de los tres años, el 2019 fue el año con mejor percepción de Cultura de seguridad del paciente, evidenciado con el mayor número de dimensiones clasificadas en fortaleza para un total de 5 de 12 . A nivel global el $64,1 \%$ del total de las respuestas fue positiva (ver tabla 2).

Según el análisis de los resultados obtenidos de la media de las respuestas del cuestionario, 4 dimensiones para el año 2019 obtuvieron medias superiores a 3,5, describiéndose con mejores valores las dimensiones de notificación de eventos relacionados con la seguridad y trabajo en equipo en el servicio, con medias de 3,92 $\pm 0,84$ y 3,92 $\pm 0,70$ respectivamente. Respecto a los resultados de diferencia de media, con un intervalo de confianza de $95 \%$, se obtuvo valores entre -0,35 a 0,70. Del total de las 12 dimensiones, únicamente Feed-back y comunicación sobre errores represento un tamaño de efecto alto (ver tabla 3 ).

Según el análisis de la correlación de la Cultura de seguridad del paciente, desarrollada entre la relación de las 12 dimensiones del cuestionario de Cultura de seguridad y la pregunta número 52 del mismo cuestionario, descrita como grado de seguridad percibida, ítem con respuesta de selección numérica de 0 a 10. Los resultados obtenidos para el año 2015 describen 9 dimensiones con grado de significancia, las dimensiones con mejores niveles para este año son: notificación de eventos relacionados con la seguridad (p: 0,000), aprendizaje organizacional/ mejora continua ( $p: 0,000)$, feed-back y comunicación sobre errores ( $p: 0,000)$ y dotación del personal (p: 0,000). Para el año 2017, las dimensiones con significancia fueron 8 de 12, manteniéndose la misma tendencia de las dimensiones con mejor significancia del año 2015. Para el 2019, según el análisis ninguna dimensión obtuvo valores significancia menor de p: 0,05. Respecto a los resultados en global, los datos guardan similitud a los años 2015 y 2017; y las dimensiones con mejor significancia son: notificación de eventos relacionados con la seguridad ( $p$ : 0,000), aprendizaje organizacional/mejora continua ( $p: 0,000$ ), feed-back y comunicación sobre errores (p: 0,000), dotación del personal ( $p: 0,000)$ y trabajo en equipo en el Servicio ( $p$ : 0,000) (ver tabla 4).

Respecto a los resultados en global, los datos guardan similitud a los años 2015 y 2017; y las dimensiones con mejor significancia son: notificación de eventos relacionados con la seguridad (p: 0,000), aprendizaje organizacional/mejora continua (p: 0,000), feed-back y comunicación sobre errores (p: $0,000)$, dotación del personal ( $p$ : $0,000)$ y trabajo en equipo en el Servicio (p: 0,000) 
Tabla 2. Estimación puntual del porcentaje de respuestas positivas, neutras y negativas en cada una de las dimensiones de la Cultura de Seguridad del paciente.

\begin{tabular}{|c|c|c|c|c|c|c|c|c|}
\hline \multirow[t]{2}{*}{ Dimensiones: } & \multicolumn{2}{|c|}{$\begin{array}{c}2015 \\
\text { (n: 172) }\end{array}$} & \multicolumn{2}{|c|}{$\begin{array}{c}2017 \\
\text { (n: 131) }\end{array}$} & \multicolumn{2}{|c|}{$\begin{array}{r}2019 \\
\text { (n: 99) }\end{array}$} & \multicolumn{2}{|c|}{$\begin{array}{l}\text { Global } \\
\text { (n:402) }\end{array}$} \\
\hline & $\%$ & \pm & $\%$ & \pm & $\%$ & \pm & $\%$ & \pm \\
\hline \multicolumn{9}{|c|}{ Notificación de eventos relacionados con la seguridad } \\
\hline Respuestas positivas & 65.3 & 0.6 & 60.3 & 0.6 & 81.9 & 0.5 & 69.1 & 0.7 \\
\hline Respuestas neutras & 26.7 & 0,6 & 28.0 & 0.4 & 15.1 & 0.5 & 23.3 & 0.4 \\
\hline Respuestas negativas & 7.9 & 0.2 & 11.7 & 0.1 & 3.0 & 0.2 & 7.4 & 0.3 \\
\hline \multicolumn{9}{|c|}{ Percepción global de seguridad } \\
\hline Respuestas positivas & 59.4 & 2.6 & 53.2 & 2.9 & 59.6 & 2.3 & 57.4 & 2.7 \\
\hline Respuestas neutras & 13.5 & 0.7 & 21.1 & 1.3 & 20.5 & 1.4 & 18.3 & 1.5 \\
\hline Respuestas negativas & 27.0 & 1.8 & 25.6 & 1.4 & 20.0 & 1.1 & 24.2 & 1.4 \\
\hline \multicolumn{9}{|c|}{ Expectativas y acciones de la supervisión del Servicio en apoyo de la seguridad } \\
\hline Respuestas positivas & 73.5 & 1.0 & 72.1 & 1.1 & 77.0 & 0.8 & 74.2 & 1.2 \\
\hline Respuestas neutras & 12.8 & 0.2 & 18.1 & 0.4 & 16.5 & 0.4 & 10.4 & 0.3 \\
\hline Respuestas negativas & 13.6 & 0.4 & 12.0 & 0.4 & 6.5 & 0.5 & 10.7 & 0.5 \\
\hline \multicolumn{9}{|c|}{ Aprendizaje organizacional/mejora continua } \\
\hline Respuestas positivas & 83.5 & 0.3 & 79.3 & 0.3 & 81.8 & 0.4 & 81.5 & 0.2 \\
\hline Respuestas neutras & 8.7 & 0.6 & 9.9 & 0.3 & 10.2 & 0.5 & 9.6 & 0.4 \\
\hline Respuestas negativas & 7.8 & 0.1 & 10.7 & 0.2 & 8.0 & 0.3 & 8.8 & 0.2 \\
\hline \multicolumn{9}{|c|}{ Trabajo en equipo en el Servicio } \\
\hline Respuestas positivas & 85.0 & 0.6 & 82.2 & 1.3 & 89.3 & 1.0 & 85.5 & 0.8 \\
\hline Respuestas neutras & 5.8 & 0.3 & 9.3 & 0.7 & 6.3 & 0.5 & 7.1 & 0.5 \\
\hline Respuestas negativas & 9.1 & 0.3 & 10.9 & 0.4 & 4.3 & 0.2 & 8.1 & 0.4 \\
\hline \multicolumn{9}{|c|}{ Franqueza en la comunicación } \\
\hline Respuestas positivas & 32.1 & 2.7 & 30.3 & 1.9 & 53.6 & 2.1 & 38.7 & 2.2 \\
\hline Respuestas neutras & 32.7 & 0.7 & 35.1 & 0.6 & 30.0 & 0.9 & 32.6 & 0.7 \\
\hline Respuestas negativas & 35.0 & 2.0 & 34.6 & 1.2 & 16.5 & 1.1 & 28.7 & 1.4 \\
\hline \multicolumn{9}{|c|}{ Feed - back y comunicación sobre errores } \\
\hline Respuestas positivas & 59.2 & 1.4 & 61.5 & 1.8 & 77.8 & 1.6 & 66.2 & 1.6 \\
\hline Respuestas neutras & 23.8 & 0.4 & 25.7 & 1.0 & 16.8 & 0.7 & 22.1 & 0.7 \\
\hline Respuestas negativas & 13.6 & 0.7 & 12.7 & 0.7 & 5.4 & 0.8 & 10.6 & 0.9 \\
\hline \multicolumn{9}{|c|}{ Respuesta no punitiva a los errores } \\
\hline Respuestas positivas & 45.1 & 1.5 & 44.8 & 1.8 & 58.9 & 1.3 & 49.6 & 1.5 \\
\hline Resp uestas neutras & 14.9 & 0.4 & 15.2 & 0.7 & 17.2 & 0.5 & 15.8 & 0.5 \\
\hline Respuestas negativas & 33.9 & 2.0 & 39.9 & 1.8 & 21.9 & 2.0 & 33.9 & 1.9 \\
\hline \multicolumn{9}{|l|}{ Dotación del personal } \\
\hline Respuestas positivas & 55.2 & 0.6 & 49.4 & 0.4 & 62.6 & 0.8 & 55.7 & 0.6 \\
\hline Respuestas neutras & 11.0 & 0.6 & 16.4 & 1.0 & 15.6 & 0.7 & 14.3 & 0.8 \\
\hline R espuestas negativas & 33.6 & 2.2 & 34.1 & 1.1 & 21.7 & 1.3 & 29.8 & 1.5 \\
\hline \multicolumn{9}{|c|}{ Apoyo de la gerencia del hospital en la seguridad del paciente } \\
\hline Respuestas positivas & 72.2 & 1.0 & 63.8 & 0.7 & 71.4 & 0.5 & 69.1 & 0.7 \\
\hline Respuestas neutras & 10.6 & 0.3 & 16.5 & 0.5 & 14.2 & 0.8 & 13.8 & 0.5 \\
\hline Respuestas negati vas & 17.0 & 0.7 & 19.3 & 0.4 & 13.7 & 0.3 & 16.7 & 0.6 \\
\hline \multicolumn{9}{|c|}{ Trabajo en equipo entre unidades/servicios } \\
\hline Respuestas positivas & 66.5 & 1.5 & 55.6 & 1.8 & 70.1 & 1.3 & 66.1 & 1.5 \\
\hline Respuestas neutras & 15.2 & 0.8 & 18.9 & 0.9 & 14.4 & 0.7 & 16.2 & 0.8 \\
\hline Respuestas negativas & 18.9 & 1.3 & 25.7 & 1.4 & 14.6 & 1.5 & 19.7 & 1.6 \\
\hline \multicolumn{9}{|c|}{ Problemas en cambios de turno y transiciones entre servicios/unidades } \\
\hline Respuestas positivas & 57.3 & 1.2 & 47.7 & 0.5 & 66.6 & 0.9 & 57.2 & 0.6 \\
\hline Respuestas neutras & 16.4 & 0.6 & 25.1 & 0.7 & 22.9 & 0.2 & 21.5 & 0.3 \\
\hline Respuestas negativas & 25.4 & 1.0 & 28.0 & 0.8 & 12.4 & 1.1 & 21.9 & 0.9 \\
\hline
\end{tabular}

Fuente: Elaboración propia. 
Tabla 3. Resultados de los puntajes de media 2015-2017-2019 y diferencia de media 2019-2015.

\begin{tabular}{|c|c|c|c|c|c|c|c|}
\hline \multirow{2}{*}{ Dimensiones } & \multicolumn{2}{|c|}{$\begin{array}{c}2015 \\
\text { (n: } 172)\end{array}$} & \multicolumn{2}{|c|}{$\begin{array}{c}2017 \\
\text { (n: } 131)\end{array}$} & \multicolumn{2}{|c|}{$\begin{array}{l}2019 \\
(n: 99)\end{array}$} & \multirow[t]{2}{*}{$\begin{array}{l}\text { Diferencia } \\
\text { de media }\end{array}$} \\
\hline & Md & DS & Md & DS & Md & DS & \\
\hline $\begin{array}{l}\text { Notificación de eventos } \\
\text { relacionados con la seguridad }\end{array}$ & 3,54 & $\pm 1,10$ & 3,36 & $\pm 1,15$ & 3,92 & $\pm 0,84$ & 0,38 \\
\hline Percepción global de seguridad & 2,81 & $\pm 0,57$ & 2,74 & $\pm 0,69$ & 2,77 & $\pm 0,53$ & $-0,04$ \\
\hline $\begin{array}{l}\text { Expectativas y acciones de la } \\
\text { supervisión del Servicio en apoyo de } \\
\text { la seguridad }\end{array}$ & 2,57 & $\pm 0,56$ & 2,46 & $\pm 0,59$ & 2,52 & $\pm 0,42$ & $-0,05$ \\
\hline $\begin{array}{l}\text { Aprendizaje organizacional/mejora } \\
\text { continua }\end{array}$ & 3,72 & $\pm 0,75$ & 3,54 & $\pm 0,83$ & 3,73 & $\pm 0,71$ & 0,03 \\
\hline Trabajo en equipo en el Servicio & 3,80 & $\pm 0,87$ & 3,66 & $\pm 0,85$ & 3,92 & $\pm 0,70$ & 0,12 \\
\hline Franqueza en la comunicación & 2,45 & $\pm 0,72$ & 2,41 & $\pm 0,78$ & 2,60 & $\pm 0,82$ & 0,20 \\
\hline $\begin{array}{l}\text { Feed-back y comunicación sobre } \\
\text { errores }\end{array}$ & 3,38 & $\pm 1,02$ & 3,40 & 0,99 & 3,90 & $\pm 0,82$ & 0,70 \\
\hline Respuesta no punitiva a los errores & 2,34 & $\pm 1,09$ & 2,37 & $\pm 1,05$ & 2,03 & $\pm 0,95$ & $-0,31$ \\
\hline Dotación del personal & 2,26 & $\pm 0,81$ & 2,27 & $\pm 0,66$ & 2,08 & $\pm 0,72$ & $-0,12$ \\
\hline $\begin{array}{l}\text { Apoyo de la gerencia del hospital en } \\
\text { la seguridad del paciente errores }\end{array}$ & 2,95 & $\pm 0,60$ & 2,84 & $\pm 0,59$ & 2,96 & $\pm 0,49$ & 0.01 \\
\hline $\begin{array}{l}\text { Trabajo en equipo entre errores } \\
\text { unidades/servicios }\end{array}$ & 2,56 & $\pm 0,55$ & 2,60 & $\pm 0,54$ & 2,49 & $\pm 0,48$ & -0.01 \\
\hline $\begin{array}{l}\text { Problemas en cambios de turno y } \\
\text { transiciones entre servicios/ } \\
\text { unidades }\end{array}$ & 2,00 & $\pm 0,94$ & 2,19 & $\pm 1,02$ & 1,65 & $\pm 0,87$ & $-0,35$ \\
\hline
\end{tabular}

Md: Media. DS: Desviación estándar. Valores de diferencia de medias. Datos menores de 0,1: Tamaño de efecto pequeño. Datos de 0,2 a 0,5: Tamaño de efecto moderado. Datos mayores de 0,5: Tamaño de efecto alta.

Fuente: Elaboración propia. 
Tabla 4. Asociación entre las dimensiones del cuestionario de Cultura de seguridad del Paciente y la pregunta 52 (grado de seguridad percibida)

\begin{tabular}{|c|c|c|c|c|c|c|c|c|}
\hline \multirow{3}{*}{ Dimensiones } & \multicolumn{8}{|c|}{ Grado de Seguridad Percibida } \\
\hline & \multicolumn{2}{|c|}{$\begin{array}{c}2015 \\
(n: 172)\end{array}$} & \multicolumn{2}{|c|}{$\begin{array}{c}2017 \\
\text { (n: } 131)\end{array}$} & \multicolumn{2}{|c|}{$\begin{array}{l}2019 \\
\text { (n: } 99)\end{array}$} & \multicolumn{2}{|c|}{$\begin{array}{l}\text { Global } \\
\text { (n: 402) }\end{array}$} \\
\hline & Coef & $\mathbf{p}$ & Coef & $\mathbf{p}$ & Coef & $\mathbf{p}$ & Coef & $\mathbf{p}$ \\
\hline $\begin{array}{l}\text { Notificación de eventos } \\
\text { relacionados con la seguridad }\end{array}$ & 0,45 & $* * *$ & 0,46 & $* * *$ & 0,02 & & 0,38 & $* * *$ \\
\hline Percepción global de seguridad & 0,00 & & 0,25 & ** & 0,07 & & 0,12 & * \\
\hline $\begin{array}{l}\text { Expectativas y acciones de la } \\
\text { supervisión del Servicio en apoyo de } \\
\text { la seguridad }\end{array}$ & 0,04 & & 0,27 & ** & 0,06 & & 0,11 & * \\
\hline $\begin{array}{l}\text { Aprendizaje organizacional/mejora } \\
\text { continua }\end{array}$ & 0,34 & $* * *$ & 0,50 & $* * *$ & 0,11 & & 0,36 & $* * *$ \\
\hline Trabajo en equipo en el Servicio & 0,24 & ** & 0,25 & ** & 0,11 & & 0,23 & $* * *$ \\
\hline Franqueza en la comunicación & 0,14 & & 0,16 & & 0,01 & & 0,12 & * \\
\hline $\begin{array}{l}\text { Feed-back y comunicación sobre } \\
\text { errores }\end{array}$ & 0,40 & $* * *$ & 0,38 & $* * *$ & 0,00 & & 0,23 & $* * *$ \\
\hline Respuesta no punitiva a los errores & 0,20 & $* *$ & 0,15 & & 0,04 & & 0.14 & $* *$ \\
\hline Dotación del personal & 0,31 & $* * *$ & 0,20 & * & 0,07 & & 0,22 & $* * *$ \\
\hline $\begin{array}{l}\text { Apoyo de la gerencia del hospital en } \\
\text { la seguridad del paciente errores }\end{array}$ & 0,15 & * & 0,16 & & 0,09 & & 0,151 & $* *$ \\
\hline $\begin{array}{l}\text { Trabajo en equipo entre errores } \\
\text { unidades/servicios }\end{array}$ & 0,25 & ** & 0,14 & & 0,04 & & 0,156 & ** \\
\hline $\begin{array}{l}\text { Problemas en cambios de turno y } \\
\text { transiciones entre servicios/ } \\
\text { unidades }\end{array}$ & 0,29 & $* * *$ & 0,40 & $* * *$ & 0,12 & & 0,31 & $* * *$ \\
\hline $\begin{array}{l}\text { P52: Pregunta 52, Grado de seguridad } \\
\text { Correlación (P52): Correlación coeficie } \\
\text { p: Significancia bilateral de Pearson: * }\end{array}$ & $\begin{array}{l}\text { ercibi } \\
\text { te de } \\
<0.05\end{array}$ & a. & $1 * *$ & & & & & \\
\hline
\end{tabular}

Fuente: Elaboración propia. 


\section{Discusión}

\section{Características de la población}

En el análisis de las características de la población participante del estudio en relación a la literatura internacional obtenida, respecto a la aplicación y medición desarrollada en estudios con base en la implementación del instrumento de análisis de la percepción de la Cultura de seguridad del paciente en hospitales "Hospital Survey on Patient Safety Culture", se describe el estudio de Pedroni, con una muestra total de 41 participantes, dividida entre personal médico (n: $21,51,2 \%$ ) y de enfermería (n: 20,48,8\%), las características obtenidas con mayor respuesta son: horas de trabajo semanales de 39 a 40 horas (n: 36, 87,8\%), y años de antigüedad en el servicio de 1 a 5 años (n: 16, 39,0\%) ${ }^{29}$. En el caso del estudio de Hori, obtuvieron una muestra de 314 participantes, el mayor número de participación fue dado por el personal de enfermería (n: 142, 45,2\%), la clasificación de horas de trabajo semanalmente con mayor respuesta fue de 40 a 59 horas (n: 140, 44,6\%) y para los años de antigüedad en el servicio la clasificación fue de 1 a 5 años (n: 123, 39,2\%) ${ }^{30}$. Para el estudio de Stewart, describiendo una muestra total de 1604 participantes, con mayor participación de enfermería (n: 802, 50,0\%), respecto a las horas de trabajo semanalmente, la clasificación con mayor frecuencia fue de 40 a 59 horas (n: 1326, $82,7 \%$ ) y de antigüedad en el servicio por años fue de 6-10 años (n: 471, 29,4\%) ${ }^{31}$. Y el estudio de Galvão, con un total de 381 participantes, con mayor participación de técnicos de enfermería (n: $132,35 \%$ ) y enfermeros ( $n: 58,15 \%)$, área de cirugía (n: $85,22,0 \%)$ y unidad de cuidado intensivo (n: 45, 12\%), horas trabajadas semanalmente con más puntuación de 20 a 39 horas (n: 252, $66,0 \%$ ) y tiempo de desempeño en su servicio 1 a 10 años (n: 112,40\%) ${ }^{32}$.

Al comparar los datos de la literatura internacional, con los resultados de este estudio respecto a las características de los participantes son similares en especial con la mayor participación del personal de enfermería, área de trabajo de Unidad de cuidados intensivos, 24-48 horas de trabajo semanales y 1 a 5 años de tiempo de desempeño en el servicio.

\section{Percepción de la Cultura de seguridad del paciente}

La Cultura de seguridad del paciente, es un sistema implementado desde hace un par de décadas con el fin de ofrecer atención en salud de calidad para todos los individuos que requieran un cuidado, la encuesta utilizada es una herramienta internacional traducida a múltiples idiomas, cuyo objetivo es medir dimensiones relacionadas con la percepción de la Cultura de seguridad del paciente. Por su importancia, su uso es muy común y por ende la evidencia literaria científica tiene un gran número de reportes, mediante la clasificación del instrumento de respuestas positivas, se logra identificar que dimensiones obtienen la etiqueta de fortaleza, oportunidad de mejora y debilidad. Con base en estudios como el de Salem, con un total de 60 reportes, las dimensiones con respuestas positivas obtuvieron porcentajes de 13 a $57 \%$, de las 12 dimensiones 6 se clasificaron como debilidad y 6 como oportunidad de mejora

33. Para el estudio de Latawi, con un total de reportes de 186, los valores de respuestas positivas están entre 23 a 85\%, las dimensiones de su estudio que se clasificaron como fortaleza fueron 4 de 12 , describiendo la dimensión de trabajo en equipo la cual obtuvo un mejor porcentaje de respuesta $(85 \%)^{34}$. 
Para el estudio de López, en el cual implementaron una estrategia para la prevención de caídas, mediante la implementación de pre-test (n: 78) y post-test (n: 40), respecto al valor de respuestas positivas que obtuvieron fue de 40,6 a $87,0 \%$, de acuerdo a esta información únicamente obtuvieron la clasificación de fortaleza en la dimensión de trabajo en equipo $(87,0 \%)^{35}$. Según el estudio de Henrietta, con un total de 37 reportes, los valores de respuestas positivas fueron de 31,0 a $77,0 \%$, con solo una dimensión clasificada como fortaleza: trabajo en equipo $(77,0 \%)^{36}$.

Respectivamente a la relación de los datos de este estudio y comparación con los anteriormente mencionados, los resultados de respuestas positivas van de 38,7 a $85,5 \%$, las dimensiones clasificadas como fortalezas son: Aprendizaje organizacional/mejora continua $(81,5 \%)$ y Trabajo en equipo en el Servicio (85,5\%). Cabe mencionar que los resultados guardan similitud con los datos de la evidencia científica internacional; enfatizando que el actual estudio en la dimensión de trabajo en equipo se identificó como fortaleza siendo percibido como excelente por parte de los trabajadores de la salud.

\author{
Cabe mencionar que los \\ resultados guardan similitud \\ con los datos de la evidencia \\ científica internacional; \\ enfatizando que el actual \\ estudio en la dimensión de \\ trabajo en equipo se identificó \\ como fortaleza siendo percibido \\ como excelente por parte de los \\ trabajadores de la salud.
}

Con base en la correlación de las 12 dimensiones del cuestionario y la pregunta 52 enunciada como grado de seguridad percibida, los datos de la evidencia científica describen estudios como el de Akogolo, con una muestra total de 384 registros, obtuvieron niveles de significancia en 11 de 12 dimensiones (p: 0,010 - p: 0,000), resaltando la dimensión de dotación al personal la cual no obtuvo nivel de significancia $(p: 0,28)^{37}$. Para el estudio de Gurkova, con un total de 1353 registros, únicamente de personal de enfermería de 4 países de Europa, obtuvieron niveles de significancia en el total de las 12 dimensiones valores de p: 0,039-p: 0,000. Describiendo la dimensión de aprendizaje organizacional/mejora continua con significancia de p: 0,000 ${ }^{38}$. Según el estudio de Zhang, con un total de 267 reportes, presentaron un total de 10 de 12 dimensiones con significancia (p: 0,033 - p: 0,000), describiendo las dimensiones de aprendizaje organizacional/mejora continua y Feedback comunicación sobre errores con significancia de p: 0,292 y p: 0,088 respectivamente, las cuales no obtuvieron buen nivel ${ }^{39}$. Y el estudio de Brborovic, con un total de 863 reportes, obteniendo significancia en 5 de 12 dimensiones ( $p$ : 0,005 - p: 0,001), enunciando la dimensión con mejor significancia: dotación personal p: 0,00140.

Respectivamente en este estudio con la literatura científica internacional, los niveles de significancia en las dimensiones medidas de la Cultura de seguridad del paciente guardan relación con datos reportados en los estudios mencionados, describiendo que para esta investigación las 12 dimensiones tuvieron significancia en los datos globales.

\section{Conclusión}

En este estudio se presentan las características y correlación de las variables de la Cultura de seguridad del paciente, por medio de la percepción de los funcionarios acerca de las diferentes dimensiones sugeridas por el instrumento Hospital Survey on Patient Safety Culture Versión Española, se observa una correlación importante, lo que genera un aporte para el abordaje de las intervenciones institucionales en pro del mejoramiento de la calidad de la atención en salud. Las preguntas con respuestas positivas permiten clasificar el total de las dimensiones con 
fin de caracterizarlas en fortalezas, oportunidades de mejora y debilidades, en relación a los resultados y al objetivo de este estudio se pudo identificar que en puntuación de global 2 dimensiones obtuvieron la clasificación de fortaleza y cabe recalcar que los datos en cuestión de años guardan similitud en sus resultados.

Se identificaron como fortalezas el aprendizaje organizacional/mejora continua y trabajo en equipo en el Servicio. Siendo resultados similares con los datos de otros estudios internacionales, en general se identifica que las dimensiones evaluadas a través del cuestionario llevan a deducir que las conductas acciones en relación a trabajo en equipo, infraestructura, clima laboral y recursos de las instituciones son medidas útiles a implementar y que permiten identificar que se está generando una cultura de seguridad del paciente en institución prestadora de servicios de salud.

Agradecimientos: Los autores agradecen la participación de los enfermeros Lucy Tatiana Afanador Afanador y Juan Pablo Quintero Ávila, en la sistematización de los datos.

Conflicto de intereses: Los autores declaran no tener ningún conflicto de intereses.

Financiación: Este proyecto no contó con fuente de financiación externa sino con rubros propios de los investigadores.

\section{Referencias}

1. Institute of Medicine. To Err Is Human: Building a Safer Health System. Washington, DC: The National Academies Press. 2000. https://doi.org/10.17226/9728

2. Dávila-Torres J. To err is human, but to not put processes in place to avoid errors from becoming fatal is inhumane. 5th International Summit of the Patient Safety Movement (PSM), California, USA, 2017. Cir Cir. 2017;85(2):101-103. https://doi.org/10.1016/j.circen.2017.03.002

3. McCallum AD, Kanagarajah S, Ford L, Patel D. To err is human: Clinical incident calls to a national travel health advice line. Vacine. 2019;37(52):7535-7538.

https://doi.org/10.1016/j.vaccine.2019.10.007

4. Nicklin W, Hugues L. Patient Safety: We've come a long way. Healthc Q. 2020;22:6-9. http://dx.doi.org/10.12927/hcq.2020.26051

5. Wainryb C, Recchia H, Faulconbridge O, Pasupathi M. To err is human: Forgiveness across childhood and adolescence. Social Development. 2019;00:1-17. https://doi.org/10.1111/sode.12413

6. Quenon JL, Vacher A, Faget M, Levif-Lecourt M, Roberts T, Fucks I, et al. Exploring the role of managers in the development of a safety culture in seven French healthcare facilities: a qualitative study. BMC Health services Research. 2020;20:517. https://doi.org/10.1186/s12913-020-05331-1

7. Atwal A, Phillip M, Morley C. Senior nurses' perceptions of junior nurses incident reporting: A qualitative study. J Nurs Manage. 2020. https://doi.org/10.1111/jonm.13063

8. Palmiere PA, Leyva-Moral JM, Camacho-Rodríguez DE, Granel-Gimenez N, Ford EW, Mathieson KM, et al. Hospital survey on patient safety culture (HSOPSC): a multi-method approach for target-language instrument translation, adaptation, and validation to improve the equivalence of meaning for cross-cultural research. BMC Nursing. 2020;19:23. https://doi.org/10.1186/s12912-020-00419-9

9. Huang CH, Wu HH, Lee YC, Van Nieuwenhuyse I, Lin CM, Wu CF. Patient safety in work environments: Perceptions of pediatric healthcare providers in Taiwan. J Pediatr Nurs. 2020;53:613. https://doi.org/10.1016/j.pedn.2020.03.005 
10.Al-Mugheed K, Bayraktar N. Patient safety attitudes among critical care nurses: A case study in North Cyprus. Int J Health Plann Mgmt. 2020;35(4):910-921.

https://doi.org/10.1002/hpm.2976

11.Chegini Z, Janati A, Afkhami M, Behjat M, Shariful-Islam SM. A comparative study on patient safety culture among emergency nurses in the public and private hospitals of Tabriz, Iran. Nurs Open. 2020;7(3):768-775. https://doi.org/10.1002/nop2.449

12.Organización Mundial de la Salud, Ministerio de Sanidad y Política Social de España. Estudio IBEAS Prevalencia de efectos adversos en hospitales de Latinoamérica. Ministerio de Sanidad y Política Social. Informes, Estudios e investigación 2009. URL: https://www.minsalud.gov.co/sites/rid/Lists/BibliotecaDigital/RIDE/DE/CA/resultados-estudio-ibeas.pdf.

13.Friganovic A, Režić S, Kurtović B, Vidmanić S, Zelenikova R, Rotim C, et al. Nurses' Perception of Implicit Nursing Care Rationing In Croatia - A CrossSectional Multicentre Study. J Nurs Manage. 2020. https://doi.org/10.1111/jonm.13002

14.Shah R, Barksfield R. Fostering patient safety through health policy innovation: A case study of implementation of the WHO Surgical Checklist in the UK. J Perioper Pract. 2020;0(0):1-5. https://doi.org/10.1177/1750458920902613

15.Abu-El-Noor NI, Abu-El-Noor MK, Zuheir-Abuowda Y, Alfaqawi M, Böttcher B. Patient safety culture among nurses working in Palestinian governmental hospital: a pathway to a new policy. BMC Health Services Research. 2019;19(1):550.

https://doi.org/10.1186/s12913-019-4374-9

16.Ministerio de la Protección Social. Guía técnica"Buenas Prácticas para la Seguridad del Paciente en la Atención en salud". Sistema Obligatorio de Garantía de Calidad en Salud. Versión 001, 2010.

URL: https://www.minsalud.gov.co/salud/CAS/Paginas/seguridad-del-paciente.aspx

17. Ministerio de Salud y Protección Social. Paquetes instruccionales: Guía Técnica "Buenas Prácticas para la seguridad del paciente en la Atención en Salud. Seguridad del Paciente y Atención Segura. Versión 2.0, 2010.

URL: https://www.minsalud.gov.co/salud/CAS/Paginas/seguridad-del-paciente.aspx

18. Betancur-Pulgarín CL, Romero-Carvajal MR, Murillo-Micolta LG, Churi-Antero Y, Angulo-Ante YN, Carmona-Carmona D. Patient Safety in a First-Level Hospital in Colombia, According to London Protocol. IntechOpen. 2020. http://dx.doi.org/10.5772/intechopen.89520

19.Sanchez AM, Betancourt A, Mantilla C, González-Vargas. Technovigilance and risk management as tools to improve patient safety in Colombian health care institutions. Rev. Ing. Bioméd. 2017;11(21):57-64. https://doi.org/10.24050/19099762.n21.2017.1173

20.Etchegaray JM, Thomas EJ, Profit J. Preoccupation with failure and adherence to shared baselines: Measuring high-reliability organizational culture. J Patient Saf Risk Manag. 2019;24(4):147-152. https://doi.org/10.1177/2516043519838185

21.Foster S. Taking the measure of safety culture. Br J Nurs, 2019;28(20):1349. https://doi.org/10.12968/bjon.2019.28.20.1349

22.Sillero-Sillero A, Zabalegui A. Safety and satisfaction of patients with nurse's care in the perioperative. Rev. Latino-Am. Enfermagem. 2019;27:3142.

http://dx.doi.org/10.1590/1518-8345.2646.3142

23.Bravo-Gómez MA, Arboleda-de-Pérez LB, Arguello DK, Becerro-Moreno X, Peñaranda-Vega M, Oyala-Naranjo DJ, et al. Cultura de seguridad en profesionales del quirófano en una institución de atención materno infantil. Rev Cubana Enferm. 2020;36(1):3155. URL: http://www.revenfermeria.sld.cu/index.php/enf/article/view/3155

24.Torijano-Casalengua ML, Olivera-Cañada G, Astier-Peña MP, Maderuelo-Fernández JA, Silvestre-Busto C. Validación de un cuestionario para evaluar la cultura de seguridad del paciente de los profesionales de atención primaria en España. Aten Primaria. 2013;45(1):21-37. http://dx.doi.org/10.1016/j.aprim.2012.07.003 
25.Gómez-Ramírez O, Arenas-Gutiérrez W, Gonzales-Vega L, Garzón-Salamanca J, Mateus-Galeano E, Soto-Gámez A. Cultura de seguridad del paciente por personal de enfermería en Bogotá, Colombia. Cienc. enferm. 2011;17(3):97-111.

https://dx.doi.org/10.4067/S0717-95532011000300009

26.Ministerio de Sanidad y Política Social de España. Análisis de la cultura sobre seguridad del paciente en el ámbito hospitalario del Sistema Nacional de Salud Español. Madrid: Ministerio de Sanidad y Consumo; 2009. URL: https://www.seguridaddelpaciente.es/es/proyectos/financiacion-estudios/percepcion-opinion/2007/analisis-cultura-seguridad/

27.Keskinova D, Dimova R, Stoyanova R. Psychometric Properties of the Bulgarian Version of Hospital Survey on Patient Safety Culture. Int J Qual Health Care. 2020.

https://doi.org/10.1093/intqhc/mzaa055

28.Salazar-Maya ÁM, Restrepo-Marín DM. Cultura de la seguridad del paciente en seis centros quirúrgicos de Antioquia. Rev Cuid. 2020;11(2):1040. https://doi.org/10.15649/cuidarte.1040

29.Pedroni VS, Gouveia HG, Vieira LB, Wegner W, Oliveira ACS, Santos MC, et al. Patient safety culture in the maternal-child área of a university hospital. Rev Gaúcha Enferm. 2020;41:20190171. https://doi.org/10.1590/1983-1447.2020.20190171

30.Hori-Okuyana JH, Freire-Galvão T, Lonardoni-Crozatti MT, Tolentino-Silva M. Health professionals' perception of patient safety culture in a university hospital in São Paulo: A cross-sectional study applying the Hospital Survey on Patient Safety Culture. Sao Paulo Med J. 2019;137(3):216-22. https://doi.org/10.1590/1516-3180.2018.0430140319

31.Stewart D, Thomas B, MacLure K, Pallivalapila A, El-Kassem W, Awaisu A, et al. Perspectives of healthcare professionals in Qatar on causes of medication errors: A mixed methods study of safety culture. PLoS One. 2018;13(9):0204801.

https://doi.org/10.1371/journal.pone.0204801

32.Galvão TF, Couteiro-Lopes MC, Carrilho-Oliva CC, Almeida-Araújo ME, Tolentino-Silva M. Patient safety culture in a university hospital. Rev. Latino-Am. Enfermagem. 2018;26:3014. http://dx.doi.org/10.1590/1518-8345.2257.3014

33.Salem M, Labib J, Mahmoud A, Shalaby S. Nurses' Perceptions of Patient Safety Culture in Intensive Care Units: A Cross-Sectional Study. Open Access Maced J Med Sci. 2019;7(21):36673672. https://doi.org/10.3889/oamjms.2019.737

34.Al-Latawi MH, Short SD, Noor-Abdulhadi NN, Murthi-Panchatcharam S, Dennis S. Assessment of patient safety culture in primary health care in Muscat, Oman: a questionnaire -based survey. BMC Family Practice. 2019;20:50. https://doi.org/10.1186/s12875-019-0937-4

35.López-Jeng C, Eberth SD. Improving Hospital Safety Culture for Falls Prevention Through Interdisciplinary Health Education. Health Promot Pract. 2019:1-8.

https://doi.org/10.1177/1524839919840337

36. Henrietta-Okafor C, Ugwu AC, Edet-Okon I. Effects of Patient Safety Culture on Patient Satisfaction With Radiological Services in Nigerian Radiodiagnostic Practice. J Patient Exp. 2018;5(4):267-271. https://doi.org/10.1177/2374373518755500

37.Akologo A, Abuosi AA, Anaba EA. A cross-sectional survey on patient safety culture among healthcare providers in the Upper East region of Ghana. PLoS ONE. 2019;14(8):e0221208. https://doi.org/10.1371/journal.pone.0221208

38.Gurková E, Zeleníková R, Friganovic A, Uchmanowicz I, Jarosová D, Papastavrou E, et al. Hospital safety climate from nurses' perspective in four European countries. Int Nurs Rev. 2019;67(2):208-217. https://doi.org/10.1111/inr.12561

39.Zhang X, Li Q, Guo Y, Lee SY. From organisational support to second victim related distress: Role of patient safety culture. J Nurs Manag. 2019;27(8):1818-1825.

URL: https://onlinelibrary.wiley.com/doi/abs/10.1111/jonm.12881

40.Beborovic O, Brborovic, Nola IA, Miloševic M. Culture of Blame-An Ongoing Burden for Doctors and Patient Safety. Int. J. Environ. Res. Public Health. 2019;16(23):4826.

https://doi.org/10.3390/ijerph16234826 